\title{
Time to Face the Fats: What Can Mass Spectrometry Reveal about the Structure of Lipids and Their Interactions with Proteins?
}

\author{
Simon H. J. Brown, ${ }^{1}$ Todd W. Mitchell, ${ }^{2}$ Aaron J. Oakley, ${ }^{1}$ Huong T. Pham, ${ }^{3}$ \\ Stephen J. Blanksby ${ }^{3}$ \\ ${ }^{1}$ School of Chemistry, University of Wollongong, Wollongong, NSW, Australia \\ ${ }^{2}$ School of Health Science, University of Wollongong, Wollongong, NSW, Australia \\ ${ }^{3}$ ARC Centre of Excellence for Free Radical Chemistry and Biotechnology, University of Wollongong, Wollongong, NSW, \\ Australia
}

\begin{abstract}
Since the 1950s, X-ray crystallography has been the mainstay of structural biology, providing detailed atomic-level structures that continue to revolutionize our understanding of protein function. From recent advances in this discipline, a picture has emerged of intimate and specific interactions between lipids and proteins that has driven renewed interest in the structure of lipids themselves and raised intriguing questions as to the specificity and stoichiometry in lipid-protein complexes. Herein we demonstrate some of the limitations of crystallography in resolving critical structural features of ligated lipids and thus determining how these motifs impact protein binding. As a consequence, mass spectrometry must play an important and complementary role in unraveling the complexities of lipid-protein interactions. We evaluate recent advances and highlight ongoing challenges towards the twin goals of (1) complete structure elucidation of low, abundant, and structurally diverse lipids by mass spectrometry alone, and (2) assignment of stoichiometry and specificity of lipid interactions within protein complexes.
\end{abstract}

Key words: Lipid-protein interactions, Structural biology, Structural lipidomics, Protein mass spectrometry

\section{Lipid-Protein Interactions}

P roteins and lipids, both integral for the function of all biological systems, are chemically distinct. A protein's three-dimensional structure and ultimately its function are defined by peptide-bonded amino acids and subsequent posttranslational modifications. In contrast, lipids are smaller molecules than proteins, but have a larger array of building blocks and linkage chemistries. Because of these inherent chemical differences, the optimal tools for de novo structural characterization differ between proteins and lipids.

Correspondence to: Simon Brown; e-mail: simonb@uow.edu.au, Stephen Blanksby; e-mail: blanksby@uow.edu.au
Lipid-protein interactions are critical for maintaining biological function in the membrane bilayer, the cytosol, and extracellular space. In the membrane, the chemical structures of lipid solvent molecules are important for the correct folding, insertion, structure, and function of membrane proteins. The interactions between enzymes and their respective lipid substrates are critical for correct substrate recognition and catalysis (e.g., lipoxygenase [1]). Additionally, lipid-protein interactions are increasingly being realized as important in maintaining cellular structure (e.g., cytoskeletal anchoring [2]), scaffolding and localization of multi-subunit protein complexes (e.g., associated kinase anchoring protein complexes [3]), and as second messengers in signal transduction (e.g., protein kinase C [4]). 
Structural characterization of lipid-protein interactions has focused primarily on the protein domains that interact with lipids [5]. Accurate chemical and structural knowledge about the lipid partner(s) is often limited; they are often treated as generic amphipathic molecules, and experimentally replaced with detergents or generic lipids. In contrast, characterization of lipid-binding proteins by biochemical and structural methods has revealed substantial knowledge about the protein sequences and structural folds involved in lipid-protein interactions.

Lipid-protein interactions display a wide range of specificities, from the largely nonspecific shell of lipids surrounding a membrane protein to highly specific interactions involved in substrate recognition, signal transduction, and membrane targeting. The most well-characterized lipid-binding domains recognize the head-group of phosphatidylinositol (PI), specifically the phosphorylation state of the head-group. For example, the FYVE domains appear to bind preferentially to PI3P [6], while $\mathrm{PH}$ domains can recognize $\mathrm{PI}(3,4) \mathrm{P}_{2}, \mathrm{PI}(4,5)$ $\mathrm{P}_{2}$, and $/$ or $\mathrm{PI}(3,4,5) \mathrm{P}_{3}$, depending on the protein sequence [5]. In contrast, BAR domains are relatively nonspecific and recognize general physical properties of the membrane (e.g., charge and curvature). At the other end of the spectrum, the steroidal factor SF-1 primarily recognizes the acyl chains of phospholipids, with selectivity for both chain length, degree of unsaturation of each acyl chain [7]. A lipid-binding protein may utilize multiple lipid-binding domains and/or membrane penetration to increase affinity and specificity for targets [5]. Multidomain, multivalent interactions can include a domain with nonspecific membrane recognition combined with a highly specific targeting domain, generating a complex with tuned membrane affinity.

\section{Structural Specificity of Interactions Between Lipids and Cytochrome c Oxidase}

Cytochrome $c$ Oxidase $(\mathrm{CcO})$ is a well-studied membrane protein in which structural studies have been substantially complemented by mass spectrometry. Structural and evolutionary conservation indicates that lipid- $\mathrm{CcO}$ interactions have survived evolution together. Comparison between the crystal structures of bovine $\mathrm{CcO}$ and bacterial $\mathrm{CcO}$ identified remarkable similarities in lipid binding. When structures of subunits I-II of the catalytic core of $\mathrm{CcO}$ from the bacterium Rhodobacter sphaeroids [8] and Paracoccus dentrificans [9] are overlaid with subunits I-II of bovine $\mathrm{CcO}$ [10], the alkyl chains of detergents in the bacterial $\mathrm{CcO}$ correspond with the positions of acyl chains of the phospholipids in the bovine $\mathrm{CcO}$ (Figure 1a, b). Evolutionary analysis of this lipidbinding site in subunits I-II demonstrated striking conservation, confirming the biological importance and co-evolution of this site [11].

Combining atomic level structural analysis with numerous enzymatic, chromatographic, and mass spectrometric techniques has further defined the selectivity of CcO-lipid interactions [10]. In this study, a remarkable observation was made; although phosphatidylglycerol (PG) molecules con-

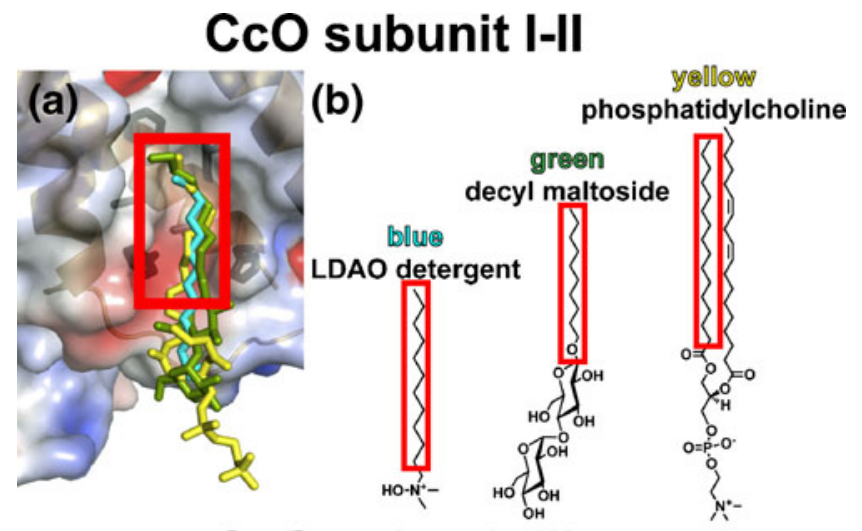

\section{CcO subunit III}

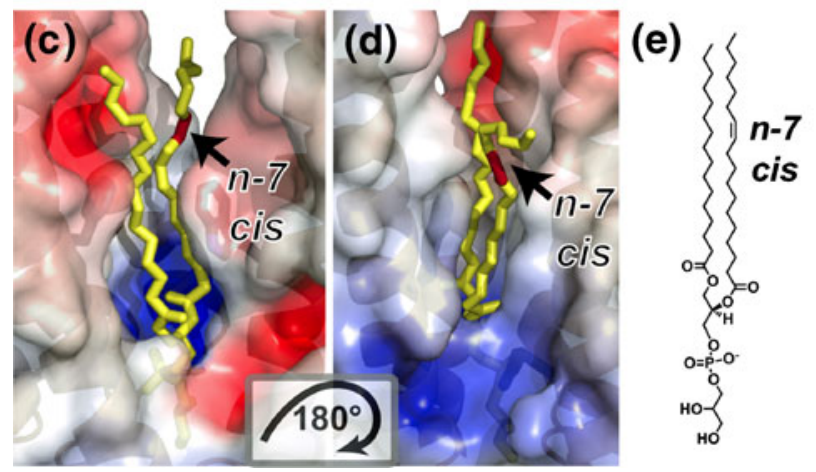

Figure 1. Conserved lipid-binding pockets of cytochrome $c$ oxidase ( $\mathrm{CcO}$ ). Bovine heart mitochondrial $\mathrm{CcO}$ (PDB ID:2DYR) is represented as a surface, colored by relative electrostatic potential, with helixes rendered as brown cartoons. (a) Phosphatidylcholine (PC) binding pocket of bovine $\mathrm{CcO}$ core subunit I bound to PC, compared with ligands found in bacterial $\mathrm{CcO}$ structures; LDAO in $\mathrm{PdCcO}$ (PDB ID: 1AR1) and decyl maltoside in RsCcO (PDB ID: 2GSM). Both LDAO and decyl maltoside as well as one acyl chain from PC all fill the same conserved pocket in respective $\mathrm{CcO}$ structures. Evolutionarily conserved acyl/alkyl-binding side chains are shown in grey. (b) Chemical structures of bound $\mathrm{CcO}$ subunit I ligands. Structures are color-coded corresponding to colors in (a); (c) and (d) two representations of a conserved pocket in $\mathrm{CcO}$ subunit III, demonstrating the uncommon $n-7$ double bond (red) of bound phosphatidylglycerol (PG) lipid determined by mass-spectrometry; (e) chemical structure of PG 16:0/18:1 (n-7)

taining $n-9$ double bonds are abundant in bovine heart mitochondrial membranes, bovine $\mathrm{CcO}$ subunit III selects almost exclusively for PG containing the $n-7$ double bond. To come to this conclusion, X-ray crystallography was combined with concurrent chemical analysis of all lipids bound to the protein in the crystals. Tenacious lipid analysis identified not only the class and acyl chains of bound lipids, but also identified the double bond position(s) and the $s n$ position (i.e., relative position of acyl chains on the glycerol backbone) in each lipid as well as the double bond stereochemistry of PG. Analysis of PG bound to the $\mathrm{CcO}$ crystals, compared with lipids of the respective membrane fraction, indicated that $\mathrm{CcO}$ subunit III is selective for both 
the stereochemistry (cis versus trans) and double bond position ( $n-7$ versus $n-9)$ in the 18:1 fatty acid of PG (Figure 1c-e). This rigorous approach to lipid structure elucidation is analogous to "bottom-up" proteomics, where the molecules are selectively broken down into their components prior to analysis and the data pieced back together to determine the original molecular structure. While bottom-up lipidomics provides significantly greater structural detail, it also requires substantially larger quantities of sample to allow for the multiple stages of sample preparation, chromatography and mass spectrometry.

\section{Structural Ambiguity of Lipids in X-ray Crystal Structures}

The importance of lipid structure has also been demonstrated for lipid-protein interactions outside of the membrane. Multiple X-ray crystal structures of the transcription factor SF-1 have been solved in complex with unknown phospholipids $[7,12,13]$. SF-1 demonstrates an interaction with a high dependence on the chemical structure of the lipid. Biochemical analysis indicates that lipid binding is only partially dependent on lipid head group class, while highly dependent on the chemical structure of the lipid acyl chains (e.g., length, degree of unsaturation and double bond position). The importance of acyl chain structure in SF-1 lipid-protein interactions is confirmed by structural analysis of the phospholipid-binding pocket, which is relatively inflexible and contacts primarily the acyl chains of the bound phospholipid. Even though the structures were solved with a variety of lipid classes, including phosphatidylethanolamine (PE), phosphatidylcholine (PC), and phosphatidylgycerol (PG), the acyl chains occupy virtually identical positions in all structures. All four structures feature ligands containing a 16-carbon acyl chain at the $s n$-2 position and two structures feature an unsaturated $s n-2$ acyl chain with the double bond located at the $n-7$ position.

SF-1 exemplifies the challenge of using X-ray crystallography as the primary tool for chemical structural identification; even high-resolution X-ray data often do not provide enough information to allow full chemical identification of unknown ligands. To demonstrate this point, we refined a SF-1 structure (PDB ID: 1YP0) with a variety of $s n-2$ acyl chain double bond isomers. These X-ray data were refined with four models containing different PE 16:0/16:1 ligands, of varying double bond positions and stereochemistry ( $n-5$ cis, $n-7$ cis, $n-9$ cis, or $n-7$ trans). An unbiased electron density map of the ligand-binding pocket of SF-1 clearly demonstrates the presence of a bound ligand with the characteristic shape of a phospholipid (Figure 2b). For each phospholipid ligand refinement, the final refinement ( $\mathrm{R}$ and Rfree) values are noted, where lower values indicate an improved refinement score (Figure $2 \mathrm{~d}-\mathrm{g}$ ). Refinement scores are the primary statistic of model quality used during model building, and provide an unbiased readout of model quality to the crystallographer. For all three cis-double bond cases,

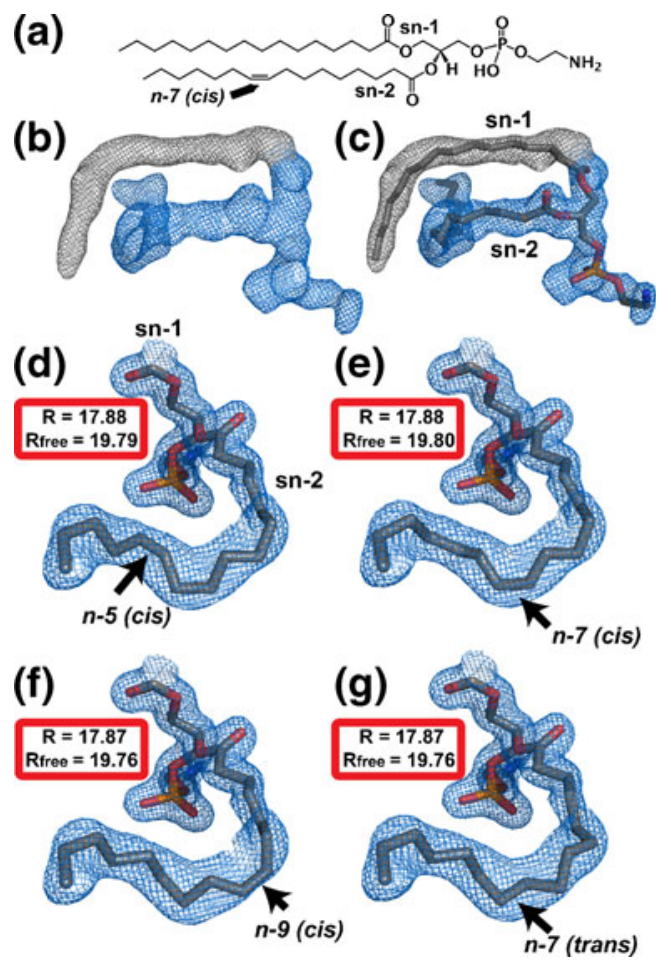

Figure 2. Demonstration of re-refinement of PDB ID:1YP0 and demonstration of acyl chain structural ambiguity. (a) Possible structure of bound PE 32:1 phospholipid. Individual acyl chain lengths, sn-positions, and double bond position were undetermined; (b) unbiased electron density map generated using a model missing PE; (c) unbiased electron density map overlaid on fully saturated 16:0/16:0 ligand from 1YPO PDB file; (d)-(g) four double bond positional and stereo

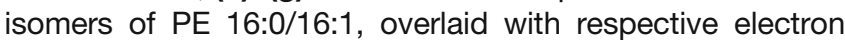
density maps. Refinement scores are shown in red box. The $s n-1$ acyl chain is removed for clarity

the variation in the double bond position did not provide substantial differences in refinement scores (Figure 2d-f). Surprisingly, even refinement of the structure with a trans double bond at the $n-7$ position did not incur a drop in the quality of refinement score (Figure $2 \mathrm{~g}$ ).

This example of SF-1 structural refinement demonstrates the limitations of lipid structure determination by crystallography. A comprehensive study of published X-ray structures has highlighted that both lipid disorder and inadequate refinement limits crystallography in structural characterization of lipids [14]. Studies combining mass spectrometry with X-ray crystallography show lipid-protein interactions are often specific and are important for protein function, yet comprehensive characterization of lipids is currently limited.

Krylova et al. observed an $[\mathrm{M}-\mathrm{H}]^{-}$anion at $\mathrm{m} / \mathrm{z} 745$ by electrospray ionization mass spectrometry (ESI-MS) analysis of an extract from SF-1 [7]. This nominal mass data is consistent with a phosphatidylglycerol with 34 carbons and two degrees of unsaturation shared by its two associated acyl chains (i.e., PG 34:2). Accurate mass measurement of this 
ion was not undertaken but could have excluded a number of commonly observed and nominally isobaric species from different lipid classes (e.g., PG O-18:1/18:1 $(\Delta \mathrm{m}=49 \mathrm{ppm})$ or PA 18:1/22:6 $(\Delta \mathrm{m}=28 \mathrm{ppm})$ ) [15]. Subsequent collision induced dissociation (CID) of this ion produced the mass spectrum shown in Figure 3 [7]. Low energy CID of ionized phospholipids are increasingly well understood [16, 17] and the product ions in this spectrum can be used to: (1) assign the acyl chains as the monounsaturated chains $16: 1(\mathrm{~m} / \mathrm{z}$ 253) and 18:1 ( $\mathrm{m} / \mathrm{z} 281)$, (2) confirm the PG classification based on the 74 Da spacing ( $m / z 491-417)$, and (3) infer that the isomer with a $16: 1$ acyl chain at the $s n-2$ position is the most abundant - and perhaps even the exclusive (see later discussion on backbone regiochemistry) - isomer present in the extract [18]. Taken together, the low energy CID spectrum can make the structural assignment of this phospholipid to PG 18:1/16:1 (where the 16:1 chain is assigned to the $s n-2$ position) consistent with the available crystallographic data.

Studies of $\mathrm{CcO}$ and SF-1 demonstrate that specific information about lipid structure in concert with protein partners can currently be obtained by mass spectrometry. The "bottom-up" approach of lipid analysis is rigorous and can give complete lipid chemical identification; however, these techniques are immensely time-consuming and have limited sensitivity because of the need for multiple stages of purification. In contrast, "top-down" lipidomic approaches in which all structure elucidation occurs on ionized and
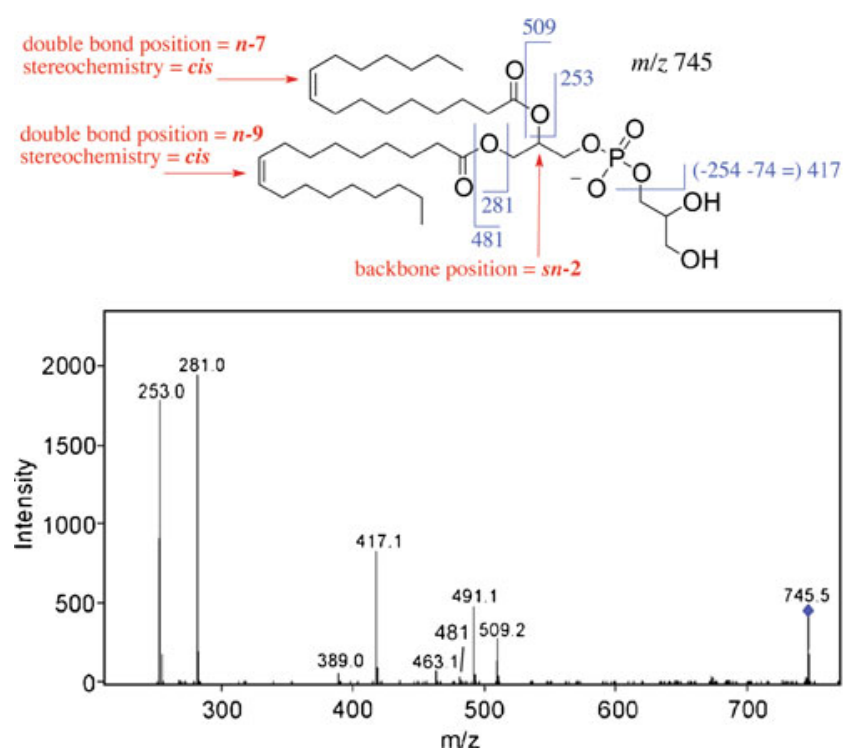

Figure 3. The collision induced dissociation mass spectrum of the $[\mathrm{M}-\mathrm{H}]^{-}$ion of PG 34:2 obtained by Krylova et al. Product ions uniquely identify this phospholipid as a phosphatidylglycerol with both $16: 1$ and $18: 1$ acyl chains as indicated by the bond cleavages shown in blue. The position and stereochemistry of the double bonds within each acyl chain (indicated in red) cannot be determined from these data. Adapted with permission from Reference [7] mass-selected lipids inside the mass spectrometer have rapid throughput and limited sample requirements. However, a current limitation of such approaches is that key structural motifs that may play critical roles in defining the specificity of lipid-protein interactions $[19,20]$ remain unresolved or ambiguous. More specifically, "top-down" techniques cannot readily determine: (1) the position of double bonds within an acyl chain; (2) the stereochemistry of double bond(s) (i.e., cis or trans); and (3) the relative position of acyl chains on the backbone of a complex lipid (i.e., sn-position on a glycerolipid). Fortunately, lipid mass spectrometry is rapidly evolving towards overcoming these limitations.

\section{Locating Carbon-Carbon Double Bonds}

Identifying the position of carbon-carbon double bond(s) in unsaturated lipids has challenged analysts for over 50 years, and this history has recently been reviewed [21]. In the context of lipid-protein interactions, methods for on-line analysis within the mass spectrometer itself are ideal as they harness the sensitivity of the mass spectrometer and even its mass-selection capabilities for lipid "purification." In this context, two distinct approaches are emerging for the identification of double bond position; namely high energy or multi-stage CID and on-line chemical derivatization approaches. Fragmentation of ionized lipids at high collision energies $(>1 \mathrm{keV})$ can bring about extensive dissociation of complex lipids including diagnostic cleavage of the carboncarbon bonds in acyl substituents. It has been known for some time that such analysis could be used to discern the position(s) of unsaturation due to preferential chain cleavage at allylic positions [22]. These original discoveries were made using sector mass spectrometers, which have now largely fallen out of favor due to their comparatively poor compatibility with contemporary ionization approaches such as ESI and MALDI. Recently, however, the commercialization of tandem time-of-flight (TOF/TOF) instruments capable of collision energies at or above $1 \mathrm{keV}$ has rekindled interest in this kind of analysis. Initial studies of triacylglycerides have demonstrated the principle of detailed structure elucidation in complex lipids using MALDI-TOF/TOF technologies [23]. While initial studies were hampered by the low mass-gating resolution (i.e., typical mass window of $4 \mathrm{Da}$ for mass selection) of TOF/TOF instruments, new developments suggest that this technical challenge is now being overcome and it is expected that these technologies will play a significant role in structural lipidomics [24]. Similar fragmentation behaviors can also be observed in the low energy regime with multiple stages of mass spectrometry $\left(\mathrm{MS}^{\mathrm{n}}\right)$. This has been carried out on ion-trap mass spectrometers [25] and more recently in combination with ion-mobility mass spectrometry to exploit time-aligned fragmentation and thus improve overall sensitivity [26]. One disadvantage of both high energy CID and multi-stage approaches is that they lead to extensive but untargeted dissociation of the lipid acyl chains such that the precursor 
ion signal is distributed over a large number of product ions: only a few of which are structurally diagnostic.

Alternative strategies for double bond position elucidation have thus emerged that incorporate more selective dissociation at the double bond by exploiting gas phase ionmolecule reactions $[27,28]$. One such approach is ozoneinduced dissociation (OzID), wherein mass-selected ions are exposed to ozone in an ion-trap mass spectrometer. In these experiments, ozone reacts selectively with the carboncarbon double bonds in unsaturated lipids giving rise to two diagnostic product ions for each double bond. An example of an OzID spectrum obtained from the $[\mathrm{M}+\mathrm{H}]^{+}$ ion of PG 16:0-18:1(9Z) (where the dash between 18:1 and $16: 0$ indicates the $s n$-position is not specified) is shown in Figure $4 \mathrm{a}$, and these data uniquely identify the $n-9$ position of the double bond within the acyl chain. OzID product ions can be predicted and thus double bond positions can be confidently assigned without reference to standard compounds [29]. This, combined with the fact that the analysis is undertaken on mass-selected ions without the need for prior fractionation and can be achieved with low sample volumes [30] suggests that it is ideally suited to the structural interrogation of lipids involved in protein binding.

\section{Assigning Backbone Regiochemistry}

For glycerolipids, the relative position of acyl chain substituents on the backbone also gives rise to isomeric variation which, as highlighted in the examples above, plays a significant role in protein binding. Observations of differences in the CID spectra of regioisomeric phospholipid anions have been reported for some time [31]. In particular, the abundance of product ions arising from the neutral loss of fatty acyl substituents can be used as an indicator of the relative backbone positions of the two acyl moieties. For example, the ions at $m / z 509$ and 481 in Figure 3 correspond to neutral losses of $\left(16: 1-\mathrm{H}_{2} \mathrm{O}\right)$ and $\left(18: 1-\mathrm{H}_{2} \mathrm{O}\right)$, respectively, and the greater relative abundance of the former is a strong indicator of its position at $s n-2$ on the glycerol backbone. These trends are not universal as the unimolecular dissociation of phospholipid anions can be influenced by phospholipid class, collision energy, and instrument geometry [32]. Additionally, given the reliance on ion abundances the presence of both isomers in differing ratios cannot be rigorously excluded. Even comparisons to synthetic standards can be challenging due to the regioimpurity of some of these formulations arising from transacylation during preparation. Ekroos and co-workers undertook a careful analysis of the phosphatidylcholine regioisomers and compared negative ion CID data with the results of the phospholipase A2 assay, which employs an enzyme to selectively hydrolyze the fatty acyl substituent at $s n-2$ [33]. This study showed that with careful calibration, the relative proportions of the two $s n$-positional isomers could be established from CID ion abundances and importantly revealed that both synthetic and biologically (a)

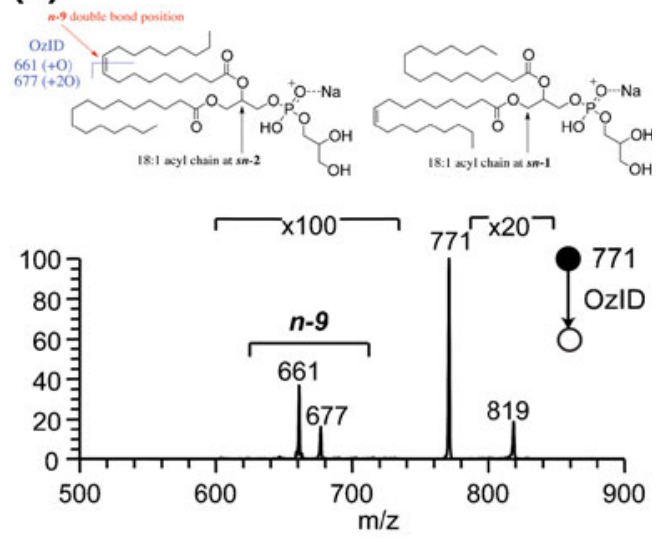

(b)
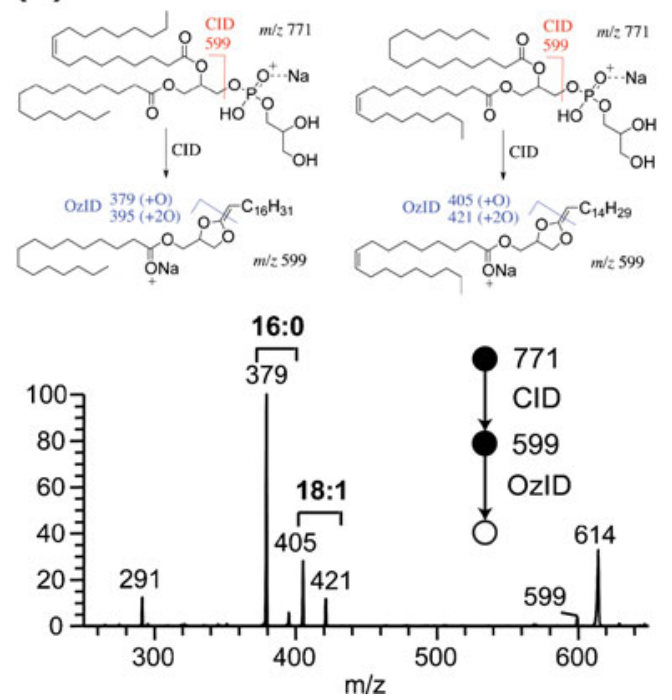

Figure 4. (a) The OzID mass spectrum of the $[\mathrm{M}+\mathrm{H}]^{+}$ions of PG 16:0-18:1(9Z) obtained on a modified linear ion-trap mass spectrometer. Product ions at $\mathrm{m} / \mathrm{z} 661$ and 677 arise from chemically induced dissociation of the carbon-carbon double bond present on the $18: 1$ acyl chain and uniquely identify the $n-9$ location of these bonds within the chain. (b) The CIDOzID mass spectrum (formally an $\mathrm{MS}^{3}$ experiment) arising from isolation of the $\mathrm{m} / \mathrm{z} 599$ product ion in the presence of ozone. Pairs of product ions at $\mathrm{m} / \mathrm{z} 379 / 395$ and $\mathrm{m} / \mathrm{z}$ 405/421 reveal the presence of both $s n$-positional isomers, PG 16:0/ 18:1 and PG 18:1/16:0, in the sample [28]

derived lipids were present as mixtures of both regioisomers. Rather than relying on ion abundances, however, a method that gives rise to unique product ions for each positional isomer would be desirable. Preliminary data suggest that combining CID with OzID may provide such a method [34, 35]. For example, $\mathrm{CID}$ of the $[\mathrm{M}+\mathrm{Na}]^{+}$adduct ion of $\mathrm{PG}$ 34:1 gives rise to an abundant product ion at $\mathrm{m} / \mathrm{z} 599$ corresponding to the neutral loss of the phosphoglycerol headgroup. Prior mechanistic studies suggest that such ions arise from nucleophilic substitution driven by the ester moiety of the $s n-2$ acyl chain [17]. The putative structure of this product ion is supported by subsequent isolation of $\mathrm{m} / \mathrm{z}$ 
599 in the presence of ozone that shows facile cleavage of the new carbon-carbon double bond and, thus, neutral loss of the $s n-2$ substituent (Figure 4b). The presence of two pairs of product ions, $\mathrm{m} / \mathrm{z} 379$ and 395 and $\mathrm{m} / \mathrm{z} 405$ and 421, corresponding to neutral losses of 18:1 and 16:1 moieties, respectively, can thus be used as evidence for the presence of two regioisomers, namely the major component of PG 16:0/18:1 and PG 18:1/16:0 as the minor isomer. Further work needs to be undertaken to establish the overall robustness and breadth of applicability of this approach.

\section{Double Bond Stereochemistry}

While most carbon-carbon double bonds in biologically derived lipids have $Z$ (cis) stereochemistry, substantial levels of $E$ (trans) isomers are often found in ruminant animals due to biohydrogenation by rumen bacteria. There is significant interest in the physiological impact of increases in dietary intake of trans fatty acids, primarily related to its correlation with increased LDL cholesterol and heart disease [36]. Not surprisingly, the gross geometric differences between isomeric lipids with cis and trans double bonds have a significant impact on molecular structure and, thus, their biophysical and biochemical properties. For example, Rauch et al. showed that the configuration of the double bond in PE 16:0/18:1 was a critical determinant of its recognition as a protein-presented antigen for $\mathrm{T}$ cells [37]. Despite its recognized importance, the ability to rapidly establish double bond stereochemistry in complex lipids presents a significant analytical challenge. Gas-chromatography (GC) of fatty acid derivatives can easily resolve and, thus, with reference to standard compounds, identify stereoisomers [38]. The application of GC to analysis of complex lipids requires rigorous fractionation of the intact lipid followed by saponification, derivatization, and subsequent chromatography. This strategy was a part of the "bottom-up" approach (described above) carried out by Shinzawa-Itoh et al., who were thus able to unambiguously assign the cis-stereochemistry of PG 18:0/18:1(11Z) bound to bovine heart cytochrome $c$ oxidase [10]. Such a multidimensional fractionation approach is extremely challenging and requires significant quantities of the lipid to be isolated. We have recently noted that ionized phospholipids bearing a trans double bond react with ozone at a greater rate than the corresponding cis-variants. This gives rise to greater peak intensities in the OzID spectra of trans versus cis lipids under the same experimental conditions [30]. While this has the advantage of allowing the analysis of a massselected and intact phospholipid, the characterization of the stereochemistry of an unknown lipid would require careful comparison to related standards, of which very few are readily available.

Conceptually, ion-mobility mass spectrometry may present a useful approach to differentiating isomeric lipids. Indeed, for other classes of molecules, separation of isomers, including chiral discrimination, has been previously demon- strated [39, 40]. Recent studies of complex lipids using conventional drift tube ion-mobility indicate that mobility is most strongly correlated with mass and thus tends to separate lipids based on class rather than isomeric variations [40, 41]. Shvartsburg et al. have shown, however, that differential ion-mobility is capable of resolving $s n$-positional isomers in diacylglycerols, which augurs well for the future discrimination of other types of lipid isomers [42]. With the commercial release of a number of different ion-mobility technologies [43, 44] further investigations of their capabilities in differentiating isomeric lipids will no doubt be undertaken. If cis and trans isomers could be rapidly distinguished by such approaches, this would represent a significant advance and provide insight not currently available from conventional tandem mass spectrometry.

\section{Evaluation of Lipid-Protein Interactions}

Many techniques to define lipid-protein interactions have been developed, each with its limitations [45, 46]. Lipidprotein overlay assays, where a membrane-bound array of lipids is probed with a protein of interest, are useful for some high affinity interactions, although the linkage of the lipid to the membrane and antibody-dependent readouts of protein binding are experimentally challenging. Surface plasmon resonance has shown promise in investigation of lipidprotein interactions, though chip-linkage chemistries and the lack of orthogonal controls limits versatility. Spin-labeled electron paramagnetic resonance (EPR) spectroscopy has proven to be a versatile technique for analysis of membrane lipids, and can reveal protein/lipid ratio, the stoichiometry of annular lipids, and information about the selectivity of lipid protein interactions [47]. EPR, however, is based on an average picture of interactions and, therefore, cannot detect heterogeneous speciation. Fluorescent labeling of lipids and/ or membrane-bound proteins have also yielded important information, especially about the dynamics of membrane systems, but are dependent on fluorescent labeling of at least one of the interaction partners. Fluorescence resonance energy transfer (FRET) is useful to probe both binding interactions and as a molecular "ruler," but again requires fluorescent labeling. Arguably, one of the most important tools in recent years for studying lipid-protein interactions has been mass spectrometry. Recent technology improvements, especially in soft ionization sources and, methods for studying noncovalent interactions, has placed mass spectrometry on the leading edge in probing the interactions between lipids and proteins and thus how structure informs about function.

\section{Mass Spectrometry for Direct Interrogation of Lipid-Protein Interactions}

For some years now, ESI (and in particular nano-ESI) mass spectrometry has been successfully employed to study the 
specificity and stoichiometry of complex protein-protein and other ligand-protein interactions [48]. To a much lesser extent, this same approach has been deployed to study lipidprotein interactions. In the case of SF-1, its affinity for a range of different phospholipids was explored by treating the protein with liposomes of different constitution and monitoring the abundance of the resulting adducts by ESI mass spectrometry [7]. More recently, Klassen and coworkers demonstrated that stable complexes between bovine $\beta$ lactoglobulin and different fatty acids could be formed by nano-electrospray ionization [49]. In this study, in-source dissociation of the delicate complexes was minimized by the addition of a high concentration of imidazole to the electrospray solution. Pleasingly, the binding constants extracted from the relative ion abundances of free and bound protein showed good agreement with traditional fluorescence-based assays. Examples such as these are, however, relatively rare. Indeed, even assemblies of membrane proteins themselves are challenging to characterize by ESI mass spectrometry because of their inherently poor solubility in ESI-compatible buffers and their susceptibility to in-source dissociation. Such difficulties can be circumvented with high surfactant concentrations that can preserve protein-protein interactions, but these conditions play havoc with ionization efficiency and give rise to large detergent aggregates. The approach taken by Robinson and coworkers has been to solubilize membrane protein-assemblies in high concentrations of nonionic detergents (e.g., $n$-dodecyl- $\beta$-D-maltoside) that also serve to protect the complex during ESI and can be subsequently striped away inside the mass spectrometer to reveal the native ensemble $[50,51]$. Importantly, when combined with careful preparation of the sample, the presence of native lipids within protein assemblies can be preserved. This has been elegantly demonstrated in the characterization of the bound lipids and their stoichiometry in two intact rotary adenosine triphosphate synthases (ATPases) from the bacteria Thermus thermophilus (Tt) and Enterococcus hirae [52]. In both instances, the intact protein assembly was observed by ESI mass spectrometry with dissociation revealing interactions of subunits with specific lipids. For example, six phosphatidylethanolamines - independently identified as PE 16:0/16:1 — were found to be associated with the $\mathrm{L}_{12}$ trans-membrane channel of the TtATPase. Tandem mass spectrometry of a subcomplex containing the $\mathrm{L}_{12}$ ring assembly revealed dissociated $\mathrm{L}$ subunits both with and without PE bound thus providing a vital clue as to the packing of lipids and proteins in this domain (Figure 5). Importantly, the specific lipids bound within each assembly were found to be the same even when different detergents were used in their preparation and, furthermore, the lipids incorporated were not the most prevalent in the cellular lipidome of the respective organisms, thus suggesting a specific structural role in tuning subunit interactions.

In parallel, Brutschy and coworkers have developed a novel alternative approach for the volatilization and ionization of biomolecular assemblies [53]. In laser-induced liquid

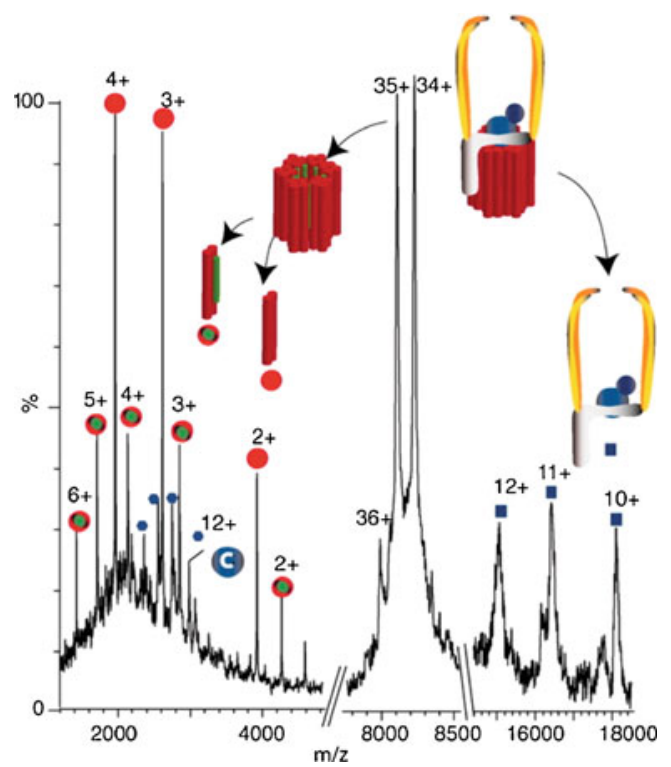

Figure 5. Tandem mass spectrum of a subcomplex from TtATPase (present in charge states $34+$ and $35+$ ) leads to disruption of the $\mathrm{L} 12$ ring and the observation of both free $\mathrm{L}$ proteins (shown as red circles) as well as proteins bound to PE 16:0/16:1 (shown as red and green circles). Reproduced with permission from Reference [52]

bead ion desorption (LILBID), molecular targets are laserdesorbed from droplets of solution containing the analyte in an essentially native environment. With no requirement for a high electric potential, LILBID is extremely tolerant of buffers, salts, and detergents and, indeed, in a recent application it was used to elucidate the changing stoichiometries of the transmembrane protein pump proteorhodopsin in the presence of different surfactants [54]. The softness of this desorption method has considerable potential for the detection of intact complexes involving weak hydrophobic bonding similar to that in many lipid-protein interactions. Conversely, the softness of the method results in ions that are often still protected by a layer of solvent, buffer, or surfactant molecules and, thus, peak broadening can hamper the determination of exact masses and, thus, stoichiometry within the complex [55]. Nevertheless, the LILBID approach appears complementary to the capabilities of ESI and both are likely to play a significant role in further unraveling the stoichiometry and specificity of lipid-protein interactions.

Finally, the ability of desorption electrospray ionization (DESI) mass spectrometry to detect large proteins and protein complexes directly from solutions [56]—so-called "liquid sample DESI" [57] — has recently been demonstrated. While for the most part, the examples provided are soluble proteins (e.g., superoxide dismutase and hemoglobin), the authors point out that this configuration has added flexibility in that solvent systems can be separately tailored for both the analyte and the ionizing spray [56]. Such a configuration may surmount some of the challenges in ESI, where the solvent system often 
represents a compromise between solubilizing the protein complex, preserving native interactions and maintaining a stable electrospray. Liquid DESI may thus provide an alternative tool for investigating lipid-protein interactions.

\section{Future Outlook}

Shevchenko has likened proteins and lipids to the knights and armies of the middle ages noting that "Knights are more visible. It's easier to write poetry about them, but in the end it's the army that wins, the collective of individuals" [58]. This metaphor captures the emerging picture of a specific and synergistic interplay between proteins and lipids in biology and highlights the significance of understanding the interactions between these two molecular classes. As we have illustrated, however, deriving this understanding challenges the classical tools of structural biology and demands new approaches.

Mass spectrometry is uniquely positioned to provide new insight into both the diversity of lipid molecular structure and the specificity of lipid-protein interactions. Examples provided herein suggest that a future in which detailed structure elucidation of lipids can be undertaken by mass spectrometry alone is not far away. Increased dissemination of such capabilities will ensure that such analyses are routinely undertaken even on the diminishingly small quantities of lipid recovered from proteins. The explicit, rather than assumed, assignment of subtle structural features provided by these new technologies (e.g., double bond position, sn-position, and stereochemistry) will become an essential complement to traditional crystallographic approaches and will go some way to rationalizing the surprising diversity of lipid molecular structures present in nature. In contrast, the direct application of mass spectrometry to the study of lipid-protein binding presents a greater technical challenge. Recent advances in the application of both traditional (e.g., nanoESI) and novel desorptionionization approaches (e.g., LILBID) to this problem, however, suggest there is also a key, complementary role for mass spectrometry in this corner of structural biology.

\section{Acknowledgments}

S.J.B. and T.W.M. are grateful to the Australian Research Council (DP0986628, DP120102922, and LP0989883) and the University of Wollongong for funding. H.T.P. is supported by a matching scholarship from the University of Wollongong and T.W.M. and A.J.O. hold fellowships from the Australian Research Council (FT110100249 and FT0990287).

\section{References}

1. Neau, D.B., Gilbert, N.C., Bartlett, S.G., Boeglin, W., Brash, A.R., Newcomer, M.E.: The 1.85 Å structure of an 8R-lipoxygenase suggests a general model for lipoxygenase product specificity. Biochemistry $\mathbf{4 8}$ (33), 7906-7915 (2009)
2. Sheetz, M.P., Sable, J.E., Dobereiner, H.G.: Continuous membranecytoskeleton adhesion requires continuous accommodation to lipid and cytoskeleton dynamics. Annu. Rev. Biophys. Biomol. Struct. 35, 417434 (2006)

3. Smith, F.D., Langeberg, L.K., Scott, J.D.: The where's and when's of kinase anchoring. Trends Biochem. Sci. 31(6), 316-323 (2006)

4. Newton, A.C.: Lipid activation of protein kinases. J. Lipid Res. 50, S266-S271 (2009)

5. Lemmon, M.A.: Membrane recognition by phospholipid-binding domains. Nat. Rev. Mol. Cell Bio. 9(2), 99-111 (2008)

6. Kutateladze, T.G.: Phosphatidylinositol 3-phosphate recognition and membrane docking by the FYVE domain. Biochim. Biophys. Acta Mol. Cell. Biol. Lipids 1761(8), 868-877 (2006)

7. Krylova, I.N., Sablin, E.P., Moore, J., Xu, R.X., Waitt, G.M., MacKay, J.A., Juzumiene, D., Bynum, J.M., Madauss, K., Montana, V., Lebedeva, L., Suzawa, M., Williams, J.D., Williams, S.P., Guy, R.K., Thornton, J.W., Fletterick, R.J., Willson, T.M., Ingraham, H.A.: Structural analyses reveal phosphatidyl inositols as ligands for the NR5 orphan receptors SF-1 and LRH-1. Cell 120(3), 343-355 (2005)

8. Qin, L., Hiser, C., Mulichak, A., Garavito, R.M., Ferguson-Miller, S.: Identification of conserved lipid/detergent-binding sites in a highresolution structure of the membrane protein cytochrome c oxidase. Proc. Natl. Acad. Sci. U. S. A. 103(44), 16117-16122 (2006)

9. Ostermeier, C., Harrenga, A., Ermler, U., Michel, H.: Structure at $2.7 \AA$ resolution of the Paracoccus denitrificans two-subunit cytochrome $c$ oxidase complexed with an antibody FV fragment. Proc. Natl. Acad. Sci. U. S. A. 94(20), 10547-10553 (1997)

10. Shinzawa-Itoh, K., Aoyama, H., Muramoto, K., Terada, H., Kurauchi, T., Tadehara, Y., Yamasaki, A., Sugimura, T., Kurono, S., Tsujimoto, K., Mizushima, T., Yamashita, E., Tsukihara, T., Yoshikawa, S.: Structures and physiological roles of 13 integral lipids of bovine heart cytochrome $c$ oxidase. EMBO J. 26(6), 1713-1725 (2007)

11. Qin, L., Sharpe, M.A., Garavito, R.M., Ferguson-Miller, S.: Conserved lipid-binding sites in membrane proteins: a focus on cytochrome c oxidase. Curr. Opin. Struct. Biol. 17(4), 444-450 (2007)

12. Li, Y., Choi, M., Cavey, G., Daugherty, J., Suino, K., Kovach, A., Bingham, N.C., Kliewer, S.A., Xu, H.E.: Crystallographic identification and functional characterization of phospholipids as ligands for the orphan nuclear receptor steroidogenic factor-1. Mol. Cell 17(4), 491-502 (2005)

13. Sablin, E.P., Blind, R.D., Krylova, I.N., Ingraham, J.G., Cai, F., Williams, J.D., Fletterick, R.J., Ingraham, H.A.: Structure of SF-1 bound by different phospholipids: evidence for regulatory ligands. Mol. Endocrinol. 23(1), 25-34 (2009)

14. Marsh, D., Pali, T.: The protein-lipid interface: perspectives from magnetic resonance and crystal structures. Biochim. Biophys. Acta Biomembr. 1666(1/2), 118-141 (2004)

15. Sud, M., Fahy, E., Cotter, D., Brown, A., Dennis, E.A., Glass, C.K., Merrill Jr., A.H., Murphy, R.C., Raetz, C.R., Russell, D.W., Subramaniam, S.: LMSD: LIPID MAPS structure database. Nucleic Acids Res. 35(Database issue), D527-D532 (2007)

16. Pulfer, M., Murphy, R.C.: Electrospray mass spectrometry of phospholipids. Mass Spectrom. Rev. 22, 332-364 (2003)

17. Hsu, F.F., Turk, J.: Electrospray ionization with low-energy collisionally activated dissociation tandem mass spectrometry of glycerophospholipids: mechanisms of fragmentation and structural characterization. J. Chromatogr. B 877(26), 2673-2695 (2009)

18. Hsu, F.F., Turk, J.: Studies on phosphatidylglycerol with triple quadrupole tandem mass spectrometry with electrospray ionization: fragmentation processes and structural characterization. J. Am. Soc. Mass Spectrom. 12(9), 1036-1043 (2001)

19. Ejsing, C.S., Sampaio, J.L., Surendranath, V., Duchoslav, E., Ekroos, K., Klemm, R.W., Simons, K., Shevchenko, A.: Global analysis of the yeast lipidome by quantitative shotgun mass spectrometry. Proc. Natl. Acad. Sci. U. S. A. 106(7), 2136-2141 (2009)

20. Schwudke, D., Hannich, J.T., Surendranath, V., Grimard, V., Moehring, T., Burton, L., Kurzchalia, T., Shevchenko, A.: Top-down lipidomic screens by multivariate analysis of high-resolution survey mass spectra. Anal. Chem. 79(11), 4083-4093 (2007)

21. Mitchell, T.W., Pham, H., Thomas, M.C., Blanksby, S.J.: Identification of double bond position in lipids: From GC to OzID. J. Chromatogr. B 877(26), 2722-2735 (2009)

22. Cheng, C.F., Gross, M.L.: Complete structural elucidation of triacylglycerols by tandem sector mass spectrometry. Anal. Chem. 70(20), 4417-4426 (1998) 
23. Pittenauer, E., Allmaier, G.: The renaissance of high-energy CID for structural elucidation of complex lipids: MALDI-TOF/RTOF-MS of Alkali Cationized Triacylglycerols. J. Am. Soc. Mass Spectrom. 20(6), 1037-1047 (2009)

24. Satoh, T., Sato, T., Kubo, A., Tamura, J.: Tandem time-of-flight mass spectrometer with high precursor ion selectivity employing spiral ion trajectory and improved offset parabolic reflectron. J. Am. Soc. Mass Spectrom. 22(5), 797-803 (2011)

25. Hsu, F.F., Turk, J.: Structural characterization of unsaturated glycerophospholipids by multiple-stage linear ion-trap mass spectrometry with electrospray ionization. J. Am. Soc. Mass Spectrom. 19(11), 1681-1691 (2008)

26. Castro-Perez, J., Roddy, T.P., Nibbering, N.M.M., Shah, V., McLaren, D.G., Previs, S., Attygalle, A.B., Herath, K., Chen, Z., Wang, S.P., Mitnaul, L., Hubbard, B.K., Vreeken, R.J., Johns, D.G., Hankemeier, T.: Localization of fatty acyl and double bond positions in phosphatidylcholines using a dual stage CID fragmentation coupled with ion mobility mass spectrometry. J. Am. Soc. Mass Spectrom. 22(9), 1552 $1567(2011)$

27. Xu, Y.H., Brenna, J.T.: Atmospheric pressure covalent adduct chemical ionization tandem mass spectrometry for double bond localization in monoene- and diene-containing triacylglycerols. Anal. Chem. 79(6), 2525-2536 (2007)

28. Thomas, M.C., Mitchell, T.W., Harman, D.G., Deeley, J.M., Nealon, J.R., Blanksby, S.J.: Ozone-induced dissociation: edlucidation of double bond position within mass-selected lipid ions. Anal. Chem. 80(1), 303311 (2008)

29. Thomas, M.C., Mitchell, T.W., Blanksby, S.J.: Online ozonolysis methods for the determination of double bond position in unsaturated lipids. In: Armstrong, D. (ed.) Lipidomics, Vol. 1, pp. 413-442. Humana Press, Buffalo (2009)

30. Poad, B.L.J., Pham, H.T., Thomas, M.C., Nealon, J.R., Campbell, J.L., Mitchell, T.W., Blanksby, S.J.: Ozone-induced dissociation on a modified tandem linear ion-trap: observations of different reactivity for isomeric lipids. J. Am. Soc. Mass Spectrom. 21(12), 1989-1999 (2010)

31. Huang, Z.H., Gage, D.A., Sweeley, C.C.: Characterization of diacylglycerylphosphocholine molecular-species by FAB-CAD-MS/MS - a general method not sensitive to the nature of the fatty acyl-groups. $J$. Am. Soc. Mass Spectrom. 3(1), 71-78 (1992)

32. Hou, W.M., Zhou, H., Khalil, M.B., Seebun, D., Bennett, S.A.L., Figeys, D.: Lyso-form fragment ions facilitate the determination of stereospecificity of diacyl glycerophospholipids. Rapid Commun. Mass Spectrom. 25(1), 205-217 (2011)

33. Ekroos, K., Ejsing, C.S., Bahr, U., Karas, M., Simons, K., Shevchenko, A.: Charting molecular composition of phosphatidylcholines by fatty acid scanning and ion trap MS3 fragmentation. J. Lipid Res. 44(11), 2181-2192 (2003)

34. Brown, S.H.J., Mitchell, T.W., Blanksby, S.J.: Analysis of unsaturated lipids by ozone-induced dissociation. Biochim. Biophys. Acta Mol. Cell. Biol. Lipids 1811(11), 807-817 (2011)

35. Stahlman, M., Pham, H.T., Adiels, M., Mitchell, T.W., Blanksby, S.J., Fagerberg, B., Ekroos, K., Boren, J.: Clinical dyslipidaemia is associated with changes in the lipid composition and inflammatory properties of apolipoprotein-B-containing lipoproteins from women with type 2 diabetes. Diabetologia 55(4), 1156-1166 (2012)

36. Brouwer, I.A., Wanders, A.J., Katan, M.B.: Effect of animal and industrial trans fatty acids on HDL and LDL cholesterol levels in humans - a quantitative review. PLoS One 5(3), e9434 (2010)

37. Rauch, J., Gumperz, J., Robinson, C., Skold, M., Roy, C., Young, D.C., Lafleur, M., Moody, D.B., Brenner, M.B., Costello, C.E., Behar, S.M.: Structural features of the acyl chain determine self-phospholipid antigen recognition by a CD1d-restricted invariant NKT (iNKT) cell. J. Biol. Chem. 278(48), 47508-47515 (2003)

38. Christie, W.W., Han, X.: Lipid analysis: Isolation, separation, identification and lipidomic analysis, 4th edn. Woodhead Publishing, Cambridge (2010)
39. Dwivedi, P., Wu, C., Matz, L.M., Clowers, B.H., Siems, W.F., Hill, H.H.: Gas-phase chiral separations by ion mobility spectrometry. Anal. Chem. 78(24), 8200-8206 (2006)

40. Trimpin, S., Tan, B., Bohrer, B.C., O'Dell, D.K., Merenbloom, S.I., Pazos, M.X., Clemmer, D.E., Walker, J.M.: Profiling of phospholipids and related lipid structures using multidimensional ion mobility spectrometry-mass spectrometry. Int. J. Mass Spectrom. 287(1/3), 5869 (2009)

41. Jackson, S.N., Ugarov, M., Post, J.D., Egan, T., Langlais, D., Schultz, J.A., Woods, A.S.: A study of phospholipids by ion mobility TOF/MS. J. Am. Soc. Mass Spectrom. 19(11), 1655-1662 (2008)

42. Shvartsburg, A.A., Isaac, G., Leveque, N., Smith, R.D., Metz, T.O.: Separation and classification of lipids using differential ion mobility spectrometry. J. Am. Soc. Mass Spectrom. 22(7), 1146-1155 (2011)

43. Giles, K., Pringle, S.D., Worthington, K.R., Little, D., Wildgoose, J.L., Bateman, R.H.: Applications of a traveling wave-based radiofrequencyonly stacked ring ion guide. Rapid Commun. Mass Spectrom. 18(20), 2401-2414 (2004)

44. Schneider, B.B., Covey, T.R., Coy, S.L., Krylov, E.V., Nazarov, E.G.: Planar differential mobility spectrometer as a prefilter for atmospheric pressure ionization mass spectrometry. Int. J. Mass Spectrom. 298(1/3), 45-54 (2010)

45. Lee, A.G.: How lipids and proteins interact in a membrane: a molecular approach. Mol. Biosyst. 1(3), 203-212 (2005)

46. Snook, C.F., Jones, J.A., Hannun, Y.A.: Sphingolipid-binding proteins. Biochim. Biophys. Acta Mol. Cell. Biol. Lipids 1761(8), 927-946 (2006)

47. Marsh, D., Horvath, L.I.: Structure, dynamics, and composition of the lipid-protein interface. Perspectives from spin-labeling. Biochim. Biophys. Acta Rev. Biomembr. 1376(3), 267-296 (1998)

48. Sharon, M., Robinson, C.V.: The role of mass Spectrometry in structure elucidation of dynamic protein complexes. Annu. Rev. Biochem. 76, 167-193 (2007)

49. Liu, L., Kitova, E.N., Klassen, J.S.: Quantifying protein-fatty acid interactions using electrospray ionization mass spectrometry. $J$. Am. Soc. Mass Spectrom. 22(2), 310-318 (2011)

50. Barrera, N.P., Di Bartolo, N., Booth, P.J., Robinson, C.V.: Micelles protect membrane complexes from solution to vacuum. Science $\mathbf{3 2 1}$ (5886), 243-246 (2008)

51. Wang, S.C., Politis, A., Di Bartolo, N., Bavro, V.N., Tucker, S.J., Booth, P.J., Barrera, N.P., Robinson, C.V.: Ion mobility mass spectrometry of two tetrameric membrane protein complexes reveals compact structures and differences in stability and packing. J. Am. Chem. Soc. 132(44), 15468-15470 (2010)

52. Zhou, M., Morgner, N., Barrera, N.P., Politis, A., Isaacson, S.C., MatakVinkovic, D., Murata, T., Bernal, R.A., Stock, D., Robinson, C.V.: Mass spectrometry of intact V-type ATPases reveals bound lipids and the effects of nucleotide binding. Science 334(6054), 380-385 (2011)

53. Morgner, N., Barth, H.D., Brutschy, B.: A new way to detect noncovalently bonded complexes of biomolecules from liquid microdroplets by laser mass spectrometry. Aust. J. Chem. 59(2), 109-114 (2006)

54. Hoffman, J., Aslimovska, L., Bamann, C., Glaubitz, C., Bamberg, E., Brutschy, B.: Studying the stoichiometries of membrane proteins by mass spectrometry: microbial rhodopsins and a potassium ion channel. Phys. Chem. Chem. Phys. 12(14), 3480-3485 (2010)

55. Hoffmann, J., Sokolova, L., Preiss, L., Hicks, D.B., Krulwich, T.A., Morgner, N., Wittig, I., Schagger, H., Meier, T., Brutschy, B.: ATP synthases: cellular nanomotors characterized by LILBID mass spectrometry. Phys. Chem. Chem. Phys. 12(41), 13375-13382 (2010)

56. Ferguson, C.N., Benchaar, S.A., Miao, Z.X., Loo, J.A., Chen, H.: Direct ionization of large proteins and protein complexes by desorption electrospray ionization-mass spectrometry. Anal. Chem. 83(17), 6468$6473(2011)$

57. Miao, Z.X., Chen, H.: Direct analysis of liquid samples by desorption electrospray ionization-mass spectrometry (DESI-MS). J. Am. Soc. Mass Spectrom. 20(1), 10-19 (2009)

58. Arnaud, C.H.: Lipids take charge. Chem. Eng. News 89(41), 15 (2011) 